\title{
Study on the Recommended Model Based on Personal Information System
}

\author{
Xiaoyu Dai \\ Computer Science \& Statistics, Letters \& Science, University of California, Berkeley, Berkeley, CA, US \\ Email: daixiaoyu0121@gmail.com
}

How to cite this paper: Dai, X.Y. (2018) Study on the Recommended Model Based on Personal Information System. Journal of Computer and Communications, 6, 40-51. https://doi.org/10.4236/jcc.2018.610004

Received: September 17, 2018

Accepted: October 26, 2018

Published: October 29, 2018

Copyright $\odot 2018$ by author and Scientific Research Publishing Inc. This work is licensed under the Creative Commons Attribution International License (CC BY 4.0).

http://creativecommons.org/licenses/by/4.0/

cC) (7) Open Access

\begin{abstract}
This paper classifies the scenario elements which affect the real-time information needs of mobile commerce users, and proposes a nomination model that integrates the user's personalized context elements. In this model, the top $\mathrm{K}$ scenarios that have the greatest impact on each user's instant information demands are calculated from the user's current scenario and historical data, thereby constructing a user personalized situation and improving it as an input condition that existing scenario-based multi-dimensional information recommendation algorithm is used for project nomination. Result/Conclusion: The improved algorithm and other three algorithms were compared by Movie lens and MBook Crossing dataset. The experimental results show that the model has higher prediction accuracy and can effectively improve user satisfaction and more effectively and solve personalized nomination issues in a mobile commerce environment.
\end{abstract}

\section{Keywords}

Personalized Model, Modern E-Commerce, Model Investigation

\section{Introduction}

In recent years, with the development of mobile communication technologies and the popularity of smart mobile devices, users are eager to obtain information and services that meet their individual needs from the mobile internet in a timely by convenient and efficient manner during the mobile process. However, because of the rapid development of information technology and its increasing content, and the limited ability of mobile devices to process information, it is increasingly difficult for people to obtain valuable information through mobile networks. In order to solve the problem of mobile information overloading and other issues, the mobile recommendation system, which has received extensive attention, has become an effective means to alleviate this problem. 
At present, scholars around the world have begun to research recommendation questions combining with user context information in mobile environment, for the characteristics that the demand of mobile commerce users' information has a strong situational dependence. Woemdl proposed a two-stage context movement recommendation model, first considering the comprehensive weight of the user state context, and then recommending when the weight reaches the set threshold [1]. Dao considered situational information into the recommendation model and adopted the combination of genetic algorithm and collaborative filtering algorithm to improve the recommendation quality [2]. Yuan Jing and others believe that the integration of mobile business situation information into the recommendation system is conducive to improving the quality of recommendation, and then propose a model for learning resource recommendation by using context information [3]. In combination with the characteristics of mobile business scenarios, Lili Zhai uses the K-means algorithm to cluster the users and adopts the collaborative filtering method to recommend, which effectively improves the mobile business information recommendation accuracy [4]. Puxiong Zhou and others proposed LBS + AR + Three-dimensional stereotype recommendation method for multilevel association rules for the characteristics of scene sensitivity in mobile information services, which introduces user context analysis to improve recommendation accuracy and quality [5]. Xiaotong Deng takes context information to users clustering, and then to improve similarity calculation [6] by social network theory to analyze user relationships and improve similarity to further improves the quality of information recommendation in the mobile commerce environment.

The above researches show that scholars at home and abroad have made certain achievements based on user situational information. However, most of them ignored the impact of difference among commerce scenarios on the needs and lacks in-depth analysis of each user's personalized situation. To this end, according to the characteristics of the mobile commerce environment, this paper classifies the scene elements to identify the main scene elements that affect the user's instant information needs, and finds the greatest $\mathrm{K}$ impact on their information needs for each user through the training set method. On the basis of the scenario elements, the personalized situation recommendation model in the mobile e-commerce environment incorporating personalized situational elements is proposed. Finally the existing context-based multi-dimensional information recommendation algorithm is improved, and the personalized situation is used as the input condition to replace the current situation information. This model provides a new idea for improving the accuracy of mobile commerce recommendation and effectively improving user experience.

\section{Context and Personalized Context Analysis in Mobile E-Commerce Environment}

\subsection{Significance and Classification of Situation}

Based on the description of the situational connotation of A. K. Dey [7], com- 
bining with the characteristics of mobile commerce, the context is defined as information about the time, location, environment and other information that affect the instant information needs of mobile commerce users.

According to the above definition, it is to classify the scene elements that affect the real-time information needs of mobile commerce users as following:

1) User context. The user context mainly refers to the user's basic context information, the behavioral state and historical preferences when using the system .Whether the user is learning or working, and he likes to exercise or stay at home during rest, these scene elements will affect distribution and selection of attention in user's mind, which is important variables that influence the outcome of the recommendation.

2) Location context. According to the description of the location context [8], it refers to the context information related to the user's location, such as the information which about the user's situation at school or home and around [8]. The user location information in this paper mainly includes: the user's city, the location type (school, home, shopping mall), the status of the people around (classmates, family, colleagues).

3) Time context. The time context mainly takes the time attribute to indicate the user's situation and its preferences, Such as the time what the user browse coupon site by IPAD (at different times of a day), the user's different demand for information resources on holidays and workdays, including that pay attention to leisure and entertainment during the holidays, but focus on work and study information in the working day.

4) Environmental context, which refers to the environmental information when the user using the recommended system, including the natural environment such as temperature and weather. The environment in which mobile commerce users are located is changeable. When designing the recommendation system, it is necessary to analyze the environmental factors to understand the real state of the use of the system, which is conducive to discovering the user's needs and changes.

5) Equipment context. At present, mobile commerce users mostly obtain information resources through mobile terminal devices (mobile phones, pads, etc.). The device information mainly includes two aspects. First, hardware device information which shows the size of the screen, affects the amount of information displayed on each page; the other includes that network information referring to the networking ways of devices that including 2G,3G, 4G and WiFi. Different network methods have different speeds. Therefore, the recommendation system needs to capture this information in time and recommend the information to users in an appropriate manner to provide better service to users.

\subsection{Personalized Context Determination}

At present, the researchers on context-based recommendation mainly focuses on the overall situation of all users as the research object, but the user's personalized situation analysis is slightly insufficient, which leads to the recommendation re- 
sult deviating from the user's instant information demand to some extent. In the process of mobile commerce information recommendation, the situation information varies from person to person. Time, location, season and temperature are very important context elements for the travel recommendation system. Some users don't think these scene elements are important but others have the opposite view, and also some users think that only time and place elements are important to them. So if we can find out the factors that affect the final selection of each user from all the situation information, which it could not only reduce the running burden of the system, but also make the prediction result more ideal [9].

Gao and others creatively proposed the concept of "individualized context". Zhou Puxiong and others borrowed this idea and expanded the connotation of individualized context. This paper is used for reference given by Zhou Puxiong. That is, personalized context refers to the largest $\mathrm{K}$ context elements that affect the instant information needs of users to be recommended [10]. This paper draws on the method of [10], which based on the user rating as a measure, firstly the training set of the user ratings matrix, and obtains the user recommendation evaluation matrix that incorporates the context elements, and then the K-optimal rating for each user. $\mathrm{K}$ context elements make up the personality of the user context, in which the $\mathrm{K}$ value is automatically set according to the optimal rating result.

The specific process of Personalized Context determination is divided into the following steps:

Step 1: Divide the user scoring matrix into training set $D$ and test set in a ratio of 80:20. Then $D$ will follow the user's current context $C=\left\{c_{1}, c_{2}, \cdots, c_{t}\right\}$ is divided into $t$ subsets, namely $D=\left\{d_{1}, d_{2}, \cdots, d_{s}\right\}$.

Step 2: Let $P_{X(U, D)}$ be the information recommendation quality evaluation standard, where $U$ is the user set, $D$ is the training set, and $X$ is the recommended algorithm used for this evaluation standard. $P_{X\left(u_{s}, d_{s}\right)}$ indicates that $u_{s}$ is the target user, and the algorithm $X$ is trained on the training set $d_{s}$ based on the context, and the user $u_{s}$ scores the information recommendation quality.

Step 3: Obtain a context-based user recommendation evaluation matrix $P_{c}$ :

$$
P_{C}=\left[\begin{array}{cccc}
P_{x}\left(u_{1}, d_{1}\right) & P_{x}\left(u_{1}, d_{2}\right) & \cdots & P_{x}\left(u_{1}, d_{s}\right) \\
P_{x}\left(u_{s}, d_{s}\right) & P_{x}\left(u_{s}, d_{2}\right) & \cdots & P_{x}\left(u_{2}, d_{s}\right) \\
\vdots & \vdots & \ddots & \vdots \\
P_{x}\left(u_{s}, d_{1}\right) & P_{x}\left(u_{s}, d_{2}\right) & \cdots & P_{x}\left(u_{s}, d_{s}\right)
\end{array}\right]
$$

Step 4: Find the context elements corresponding to the $\mathrm{K}$ optimal values in the corresponding row for each user through step 3 , and compose the context to set $C^{\prime}=\left(C_{1}^{\prime}, C_{2}^{\prime}, \cdots, C_{K}^{\prime}\right)$, which is user's personalized context.

\section{Model Construction}

At present, most of the context-based recommendation systems are information recommendations based on the user's overall context, but ignoring the in-depth 
analysis of each user's personalized context. In view of this, this paper introduces user personalization based on existing context recommendation research that a personalized context recommendation model for mobile e-commerce environment is constructed. The recommendation process of the model begins by generating user's personalized situation through the user's current context and multi-dimensional historical rating data, and then jointly taking rating data obtained from the multi-dimensional rating data warehouse to get a multidimensional data $\mathrm{U}$ (user) $\times \mathrm{I}$ (project) $\times \mathrm{C}^{\prime}$ (personalization context) $\times \mathrm{R}$ (rating) based on personalized context $C^{\prime}$ and as input to select relevant rating data condition, based on these selected rating data combined with the multi-dimensional information recommendation algorithm based on personalized situation proposed in this paper, the final recommendation list $\{\mathrm{I} 1, \mathrm{I} 2, \mathrm{I} 3, \cdots\}$ is obtained.

\subsection{Personalized Context Analysis Module}

Its main function is to find out $\mathrm{K}$ scene elements that have the greatest impact on users' instant information needs, which means the user's personalized scenes, current situation and multi-dimensional rating data warehouse are the basis for the system to generate user personalized context [11]. The user's current context can be obtained through a mobile device with built-in sensors and RFID. The multidimensional historical rating data of the project accepted by the users of multidimensional rating data warehouse stores. Each rating data first extracts the main attributes of each dimension document of the user, project and context, and then combines the rating data saved by the system to form a multidimensional historical rating data and stores it. The multidimensional rating data warehouse is an important basis for determining the user's personalized context and also a key data source for multi-dimensional information recommendation.

\subsection{Multidimensional Information Recommendation Module}

It is the most important component of the mobile e-commerce personalized context recommendation model. This module first uses multi-dimensional historical rating data and user personalized contexts obtained from the personalized context analysis module to gain multi-dimensional data $\mathrm{U} \times \mathrm{I} \times \mathrm{C}^{\prime} \times \mathrm{R}$ based on personalized contexts Based on the proposed the multi-dimensional information recommendation algorithm of the particular scene, it is recommended for the project.

\subsection{User and Recommendation System Interaction Module}

The module is an integral part of the recommended model in this article. As the object of the mobile commerce recommendation service, the users submits recommendation request to the recommendation system, and obtains the recommendation result provided by the system, that make the users to experience and gives a rating [12] for the recommendation result. At this time, the user's current situation and the recommendation resulting rating will be transmitted 
instantly to the multidimensional rating data warehouse, which will update the contexts and ratings.

\section{Multidimensional Information Recommendation Algorithm Based on Personalized Contexts}

\subsection{Algorithm Design Idea}

In this paper, the mobile user's current personalized situation C' is used instead of the current situation $\mathrm{C}$ as the filtering condition, and the rating data segment contained in the scene elements that have less influence on the user's score is excluded to select scene sufficiently similar to the current personalized situation $\mathrm{C}^{\prime}$, in order to score the data segment and determine if there is a certain use for this data segment which the user has different scores for the same project in different contexts, and then make the traditional two-dimensional recommendation algorithm to perform score prediction. The improved algorithm [13] of the existing multi-context recommendation algorithm based on the context can not only reduce the burden of system operation, and also effectively improve the quality of the recommendation.

When the multi-dimensional data is selected to use the personalized context $C^{\prime}$, in the actual situation, it may lead to an extremely sparse scoring matrix if in over-explicit situation. Therefore, the selection condition used in this paper is C' $\in \mathrm{SC}^{\prime}, \mathrm{SC}^{\prime}$ is the superior situation to which the personalized situation $\mathrm{C}^{\prime}$ belongs, such as C" = "girlfriend", then SC" = "friend".

\subsection{Algorithm Process Description}

Input: $\mathrm{MR}$ (Multidimensional Ratings), $\mathrm{MR}=\left\{\left(\mathrm{U}_{p} \mathrm{I}_{k}, \mathrm{C}_{p} \mathrm{R}_{U j, I, C)}\right\} ; j=1, \cdots, m ; k\right.$ $=1, \cdots, n$ (total of $m$ users, $n$ items). $\mathrm{C}_{1}$ is a historical context in multidimensional scoring data, $l=1, \cdots, q$.

User's current personalized context C', target user U1.

Output: Target user $\mathrm{u}_{1}$ predicted rating for project $\mathrm{I}_{1}$ in the current personalized context $\mathrm{C}^{\prime}$ are $\mathrm{Pu}_{1}, \mathrm{I}_{1}, \mathrm{C}^{\prime}$.

The specific process of the algorithm is divided into the following steps:

Step 1: Filter the MR to filter out the rating data set MD that is sufficiently similar to the user's current personalized context C'.

Step 2: Determine if step 1 is difficult to construct due to data sparsity the score data segment MD with the context is sufficiently similar to the current personalized context C'. If doesn't exist, directly execute the next step. Otherwise, SC' is used instead of C' as the selection condition to select the score data segment, $\mathrm{SC}^{\prime}$ is the personality the superior situation to which context $\mathrm{C}^{\prime}$ belongs.

Step 3: Determine whether there is a situation in the MD that different users have different scores for the same item in different contexts. If the problem does not exist, go directly to step 4 . Otherwise, the user is in different contexts which different scores are aggregated to get the final result of the user for a project rat- 
ing. That is : $R_{U_{j}}, I_{k}=A V G\left(U_{j}, I_{k}, C_{l}\right)$, which guarantees the user get a unique rating value for the same item, resulting in the final score data segment $\mathrm{MD}$ after the personalized context filtering.

Step 4: Calculate the similarity of the MD using the traditional two-dimensional recommendation algorithm:

$$
\operatorname{Sim}\left(u_{1}, U_{j}\right)=\frac{\sum_{S \in I\left(S_{u_{1}}, U_{j}\right)}\left(R_{u_{1}, s}-\overline{R_{u_{1}}}\right)\left(R_{U_{j^{s}}}-\overline{R_{U_{j}}}\right)}{\sqrt{\sum_{S \in S_{u_{1}}, U_{j}}\left(R_{u_{1}, s}-\overline{R_{u_{1}}}\right)^{2}} \sqrt{\sum_{S \in S_{u_{1}}, U_{j}}\left(R_{U_{j^{s}}}-\overline{R_{U_{j}}}\right)^{2}}}
$$

in where, the average value of all items rating by the target user $\mathrm{u} 1 \mathrm{in} \mathrm{MD}$ is represented by $\overline{R_{u_{1}}}$; The average value of all items rating by the target user $U_{j}$ took as $R_{U j^{\circ}}$

Step 5: Predict the items rating to be recommended

$$
P_{u_{1}, I_{i}^{\prime} C}=\overline{R_{u_{1}}}+k \sum_{j=1}^{N}\left(R_{U_{j}, I_{i}}-\overline{R_{U_{j}}}\right) \times \operatorname{sim}\left(u_{1}, U_{j}\right)
$$

in where $k=\frac{1}{\left.\Sigma_{U_{i} \in U^{\prime}}\right|_{s i m}\left(u_{1}, U_{j}\right)}$.

The set of $N$ users most similar to the recommended user $\mathrm{u}$ is denoted as $\mathrm{u}$ '. The $N$ value is usually preset by the system.

Step 6: Rank them in descending order according to the size of the rating value, and recommend TOP-N as the recommended set to target users.

\section{Experimental Results and Analysis}

\subsection{Experimental Data Sets and Evaluation Criteria}

In order to verify the prediction effect of the multi-dimensional information recommendation algorithm based on personalized contexts, the Movie lens data set [14] provided by the University of Minnesota was adopted in this paper, and the numerical experiment [15] is carried out on the basis of Book rating data provided by Book-Crossing, the real data set of MBook Crossing constructed by integrating the reasonable scenario was generated, and the rating data is randomly divided into training set and test set according to the ratio of 80:20, and the context-based collaborative filtering algorithm, the classic Slope One algorithm and the dimension-based input are selected [16], which is a comparative analysis of the contextual multidimensional information recommendation algorithm [17].

The Movie lens data set available publicly at the University of Minnesota, USA, provides 100,000 film scores and is widely used in recommended empirical testing. The data set contains user context information such as user gender, age, occupation, etc. Time contexts (such as working days, statutory holidays, etc.) can be obtained indirectly through unix timestamp data, and the cleaned and quantified Movie lens data set can be obtained indirectly [18]. User preferences can be better used directly by the recommended algorithm. The Book-Crossing dataset is a collection of 278,858 user pairs collected by Cai-Nicolas Ziegler from 
the Book-Crossing book community using a crawler 271,379 behavioral information carried out in this book. Based on the Book-Crossing data set, the data set MBook-Cross constructed by reintegrating the situational information, contains basic information about user ID, location, age, time (morning, noon, afternoon, evening), location (office, home, school, restaurant), environmental context information (sound environment, space), status information (entertainment, work, study), etc.

This paper uses the average absolute error widely used in the recommended quality test method MAE [19] and P(U)@N [20] are used as metrics. The MAE measures the accuracy of the recommendation results by calculating the deviation between the predicted rating and the actual rating [21], while $\mathrm{P}(\mathrm{U}) @ \mathrm{~N}$ represents the Top- $\mathrm{N}$ recommended column generated by the user. The ratio of the number of items in the table that meets their needs to $\mathrm{N}$ is calculated as follows:

$$
\begin{gathered}
\mathrm{MAE}=\frac{\sum_{i=1}^{N}|p i-q i|}{N} \\
\mathrm{P}(\mathrm{u}) @ \mathrm{~N}=\frac{\text { relevanititemin top } n \text { items for } u}{N}
\end{gathered}
$$

\subsection{Experimental Results and Analysis}

1) Personalized Context Determination Training According to the Specific Steps in Section 1.2

The user ratings on the set are trained to obtain a context-based user recommendation evaluation matrix.

The context-based user recommendation evaluation matrix for the Movie lens data set is:

Gender Time ... Preference

$$
P_{C}=\left[\begin{array}{cccc}
4.868 & 4.808 & \cdots & 3.855 \\
3.701 & 2.684 & \cdots & 4.188 \\
\vdots & \vdots & \ddots & \vdots \\
4.792 & 0 & \cdots & 4.325
\end{array}\right]
$$

The context-based user recommendation evaluation matrix for the MBook Crossing data set is:

Time Position ... Status

$$
P_{C}=\left[\begin{array}{cccc}
4.875 & 4.781 & \cdots & 2.956 \\
3.774 & 1.885 & \cdots & 4.809 \\
\vdots & \vdots & \ddots & \vdots \\
2.972 & 4.570 & \cdots & 3.631
\end{array}\right]
$$

The rows in the matrix represent the individual users, and the columns represent the corresponding contexts. Each value represents the user's rating of the information recommendation quality of the user under ct. If the system sets $K=5$, the five highest scores in each row are composed User's personalized context C'. 
2) Analysis of Prediction Results

The algorithm proposed in this paper is compared with the context-based collaborative filtering algorithm, the classic Slope One and the input-contextual multidimensional information recommendation algorithm based on dimensionality reduction. The specific comparison results are shown in Figures 1-4.

As shown in Figure 1, due to the lack of some external context information in the Movie lens dataset, the rating data segments with the selected contexts and the current personalized context C' have certain sparsity, resulting in the large volatility in MAE values of the other three algorithms, and when the number of near neighbors is less than 0 - 150, the MAE values of the algorithms are large, but with the increase of the number of near neighbors, the MAE value shows a trend of decreasing and tends to be stable, less than the other three algorithms.

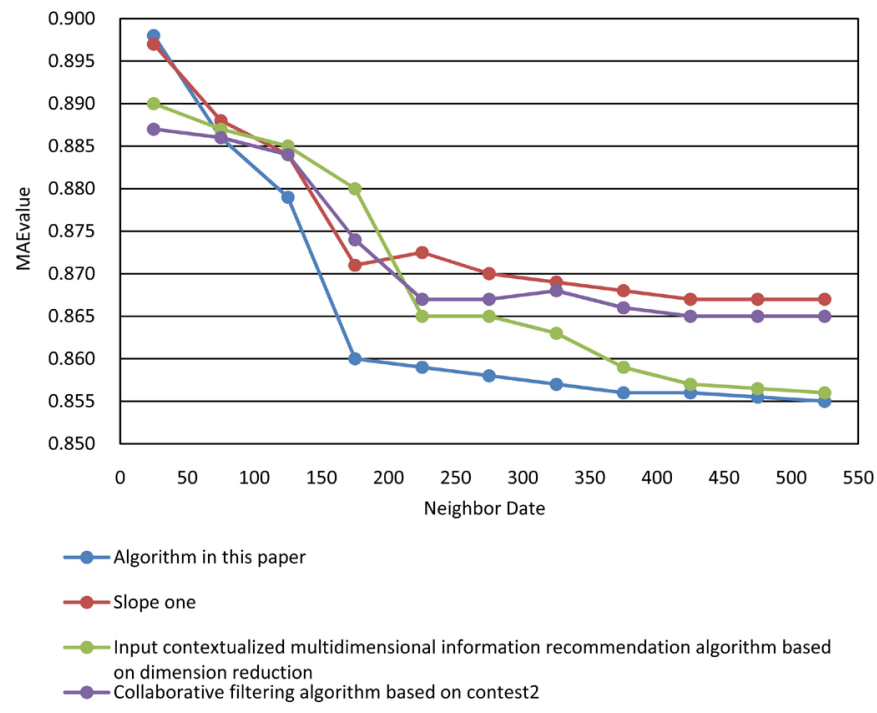

Figure 1. Comparison of MAE results of 4 algorithms on the Movie lens data set.

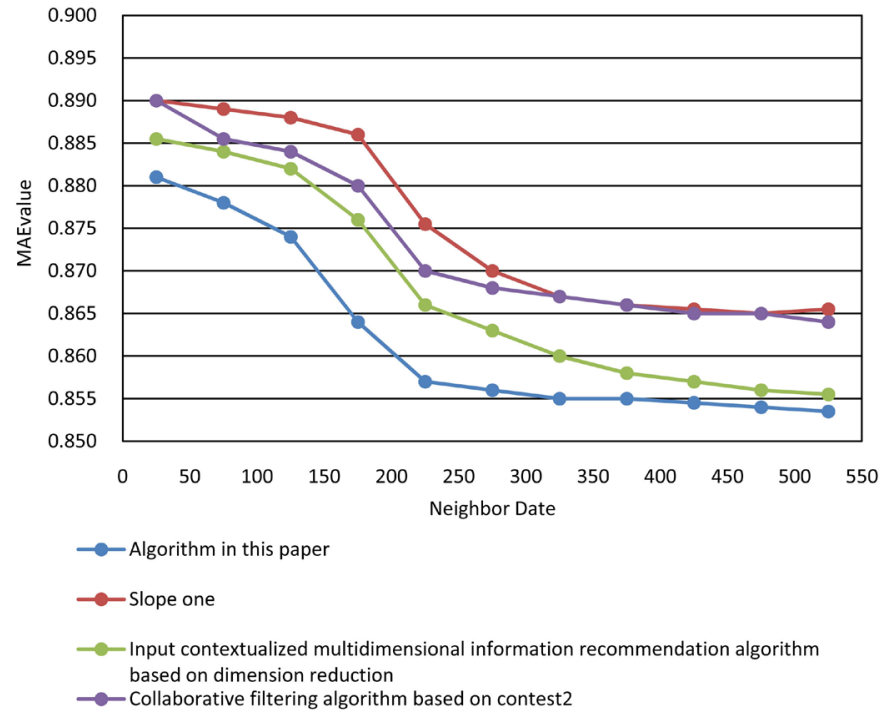

Figure 2. Comparison of MAE results of 4 algorithms on the MBook crossing data set. 


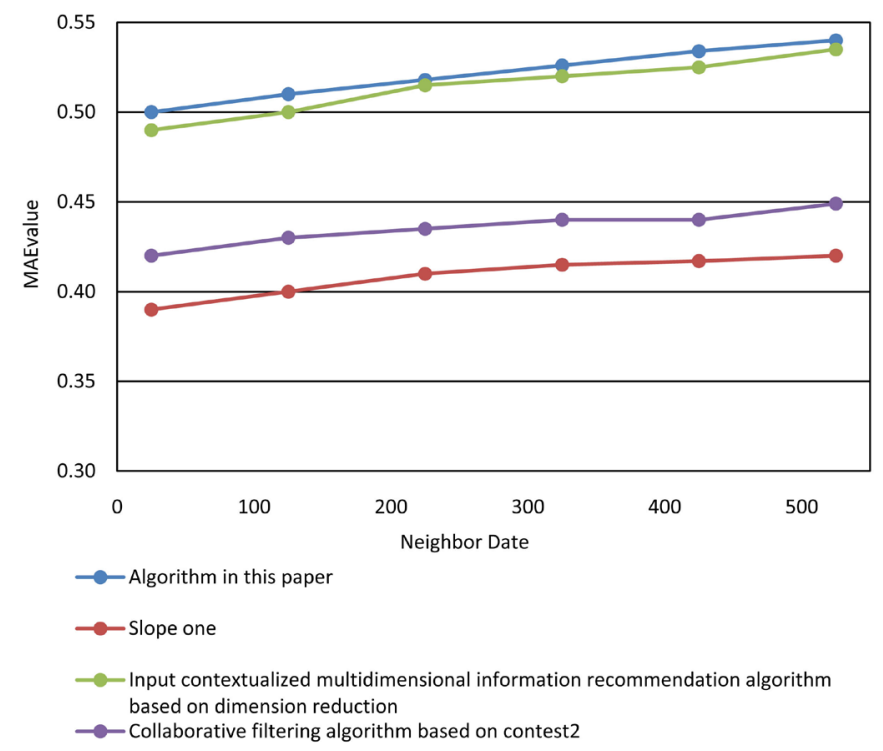

Figure 3. The $\mathrm{P}(\mathrm{U}) @ \mathrm{~N}$ comparison results of 4 algorithms on the Movie lens data set.

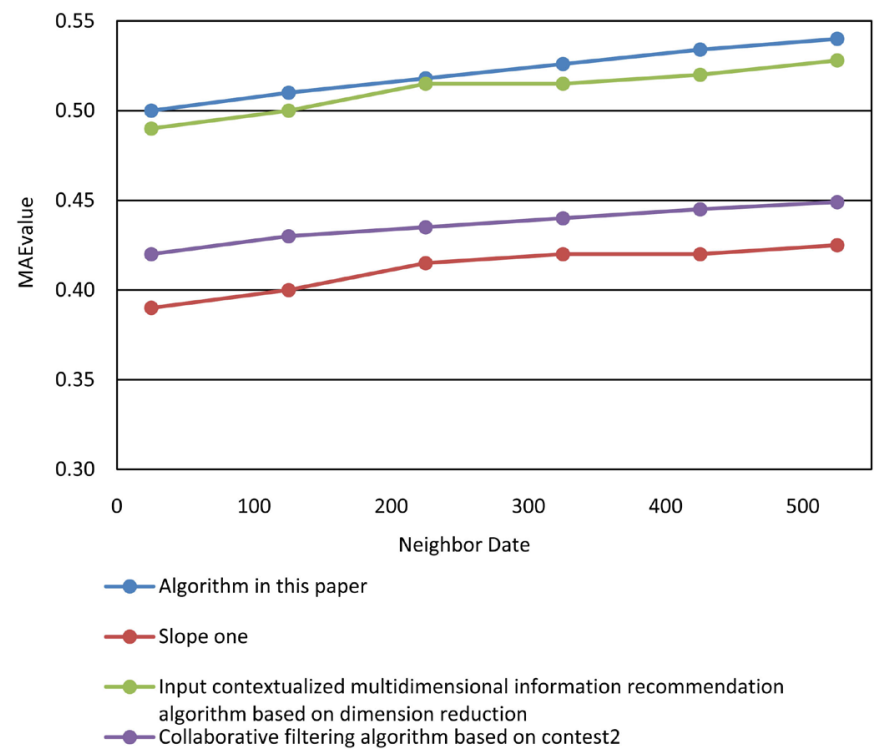

Figure 4. The $\mathrm{P}(\mathrm{U}) @ \mathrm{~N}$ comparison results of the four algorithms on the MBook crossing data set.

In Figure 2, the MAE values of the four algorithms show a rapid downward trend with the increase of the number of neighbor users, and the MAE value of the algorithm is the smallest, the prediction have the highest accuracy in this paper, which indicates that the multi-dimensional information recommendation algorithm based on the personalized situation is better than the information recommendation algorithm what was neglected or only considered the user's overall situation, because the differences in the impact of mobile commerce contexts on different user information needs, and it can be seen from the results that considering the impact of personalized contexts on users can effectively improve the quality of recommendations [21]. 
As can be seen from Figure 3 and Figure 4, when selecting the number of different neighbors in the $\mathrm{P}(\mathrm{U}) @ \mathrm{~N}$ performance comparison, the collaborative filtering algorithm considering the overall situation information and the input context-based recommendation algorithm based on dimensionality reduction are more accurate than the Slope One algorithm. The proposed algorithm is more effective and has a higher $\mathrm{P}(\mathrm{U}) @ \mathrm{~N}$ accuracy rate, indicating that the algorithm in this paper is reasonable considering individualized contexts in the case of elements. It has a higher recommendation quality and can better meet the mobile instant information needs of business users.

\section{Conclusion}

Based on context information recommendation, there is a lack of in-depth analysis of user personalized contexts on the existing research. This paper proposes a personalized context recommendation model in mobile e-commerce environment, and improves the multi-dimensional information recommendation algorithm based on context. The model calculates the personalized context of each user firstly, and then replaces the current context with each user's personalized context for multi-dimensional information recommendation. Since this model first determines, the context elements that have the greatest impact on user recommendation, it is more in line with the fact that humans have different needs in different context. Finally, experiments are carried out on the data sets of Movie lens and MBook Crossing, and the results show that the model and improved algorithm proposed in this paper have higher prediction accuracy, better recommendation quality, and can effectively solve the personalized recommendation problem in the mobile commerce environment. The future research will combine the social network theory and long tail theory with the method in this paper to further improve the quality of algorithm recommendation.

\section{Conflicts of Interest}

The author declares no conflicts of interest regarding the publication of this paper.

\section{References}

[1] Woerndl, W., Vico, D.G. and Bader, R. (2016) A Study on Proactive Delivery of Restaurant Recommendations for Android Smartphones. http://pema2011.cs.ucl.ac.uk/papers/pema2011_vico.pdf

[2] Dao, T.H., Jeong, S.R. and Ahn, H. (2012) A Novel Recommendation Model of Location-Based Advertising: Context-Aware Collaborative Filtering Using GA Approach. Expert Systems with Applications, 39, 3731-3739. https://doi.org/10.1016/j.eswa.2011.09.070

[3] Jing, Y. and Jiao, Y. (2009) Personalized Recommendation of Learning Resources Based on Context Information. Intelligence Theory and Practice, 32, 116-119.

[4] Zhai, L., Xing, H. and Zhang, S. (2016) Research on Collaborative Filtering Recommendation of Mobile E-Commerce Based on Situation Clustering Optimization. Information Theory \& Practice, 39, 106-110. 
[5] Zhou, P. and Tao, M. (2012) Research on Situation-Sensitive Personalized Information Recommendation System in Mobile Network Environment. Library and Information Service, 56, 80-81.

[6] Deng, X., Chun, J., Han, Q., et al. (2013) Collaborative Filtering Recommendation Model Based on Situation Clustering and User Rating. Systems Engineering. Theory \& Practice, 33, 2945-2946.

[7] Dey, A.K. (2001) Understanding and Using Context. Personal and Ubiquitous Computing Journal, 5, 4-7. https://doi.org/10.1007/s007790170019

[8] Zhai, L., Jing, W. and He, X. (2015) Software Industry Virtual Machine Group Cooperation Competition Mechanism. Science Press, Beijing, 182-183.

[9] Wen, G. and Wu, Z. (2009) Research on Collaborative Recommendation Based on Personalized Situation and Project. Journal of Southeast University: Natural Science Edition, 39, 29-30.

[10] Zhou, P., Xue, W. and Zhao, W. (2015) Multi-Agent Based on Personalized Situation Information Recommendation Research. Intelligence Journal, 34, 182-183.

[11] Jun, Y. (2011) Research on Multi-Dimensional Information Recommendation Based on Situational Awareness. Wuhan University, Wuhan.

[12] Jun, Y., Wang, H. and Ai, D. (2014) A New Method of Multidimensional Information Recommendation Based on Output Situationalization. Information Science, 32, 126-132.

[13] Jun, Y., Zan, M. and Ai, D. (2013) Research on Input and Context Multidimensional Information Recommendation Based on Dimensionality Reduction. Information Theory \& Practice, 36, 64-65.

[14] Xu, H.L., Wu, X., Li, X.D., et al. (2009) Comparison Study of Internet Recommendation System. Journal of Software, 20, 350-362. https://doi.org/10.3724/SP.J.1001.2009.00350

[15] Xun, H., Meng, X., Zhang, Y., et al. (2014) A Recommendation Algorithm for Fusion Project Features and Mobile User Trust Relationship. Journal of Software, 25, 1824-1825.

[16] Kim, J., Lee, D. and Chung, K. (2014) Item Recommendation Based on Context-Aware Model for Service. Multimedia Tools and Applications, 71, 855-872. https://doi.org/10.1007/s11042-011-0920-0

[17] Cyohulyad, R. and Leray, P. (2014) A Personalized Recommender System Relational Model and Users Preferences. Procedia Computer Science, 35, 1063-1067.

[18] Xu, F.W., Meng, X.W. and Wang, L.C. (2011) Collaborative Filtering Recommendation Algorithm Based on Context Similarity of Mobile Users. Journal of Electronics \& Information Technology, 33, 2785-2788. https://doi.org/10.3724/SP.J.1146.2011.00384

[19] Hou, S., Sun, L. and Jin, X. (2018) Method and System for Protecting Personal Information Based on Mobile Terminal and the Mobile Terminal. US20180053013.

[20] Ju, J.Y. and Dongsim, K. (2017) A Study of Outcomes Utilizing K-MOOC Credit Based on the Information System Success Model. Journal of Educational Studies, 48, 95-113. https://doi.org/10.15854/jes.2017.06.48.2.95

[21] Li, E. (2017) Research on the Selection of Personal Information Security Model Based on the Fuzzy Prospect Theory. Journal of Chongqing University of Science \& Technology, 3, 124. 